\title{
Advancing the Optical Feed Back Concept: Grating Enhanced External Cavity Diode Laser
}

\author{
A. Wicht ${ }^{1}$, P. Huke ${ }^{2}$, R.-H. Rinkleff ${ }^{2}$ and K. Danzmann ${ }^{2,3}$ \\ ${ }^{1}$ Institut für Experimentalphysik, Heinrich-Heine Universität Düsseldorf, Universitätsstr. 1, D-40225 Düsseldorf, Germany \\ ${ }^{2}$ Institut für Atom- und Molekülphysik, Universität Hannover, Callinstr. 38, D-30167 Hannover, Germany \\ ${ }^{3}$ Max-Planck Institut für Gravitationsphysik, Callinstr. 38, D-30167 Hannover, Germany
}

Received October 18, 2003; revised version received August 9, 2004; accepted August 20, 2004

PACS numbers: 42.55.Px, 42.60.By, 42.40.Eq

\begin{abstract}
We present theoretical and experimental investigations on a new optical feedback concept for single mode diode lasers. It is based on the optical feedback from a resonant external cavity coupled to the diode laser through the 1 st order diffraction of a grating. With a single mode diode laser operating at $852 \mathrm{~nm}$ we demonstrate a short term line width of less than $60 \mathrm{kHz}$, an overall tuneability in excess of $36.4 \mathrm{~nm}$ and a continuous tuning range in excess of $45 \mathrm{GHz}$, both at constant diode temperature.
\end{abstract}

\section{Introduction}

In the recent years tunable laser systems became essential for fields like spectroscopy and telecommunications. The demand for highly reliable, low cost, and easy-to-use laser systems made tunable diode laser systems a favorable design. Additionally these cheap sources of coherent radiation offer large modulation bandwidth, a large continuous tuning range and narrow line width when stabilized and controlled via optical feedback.

To avoid confusion about the terms used throughout the paper, we want to give a precise definition of two complementary concepts:

1. The extended cavity diode laser concept [1] is based on optical feedback from a grating that selects the wave length. The 1st order output beam is either directly fed back from the grating, Littrow-setup, or retro-reflected from an additional mirror following the grating, Littman-setup. This concept shows an overall tuning range of several $\mathrm{GHz}$. The line width ranges typically from $100 \mathrm{kHz}$ to several $\mathrm{MHz}$ depending on the line density of the grating and on the type of the diode laser.

2. The second concept uses an external cavity [2], commonly called diode laser with resonant optical feedback, see also [3]. A small fraction of the radiation coming from the diode laser is coupled into a resonant Fabry-Perot cavity. The resonant light reflected by the cavity is fed back to the diode laser. The finesse of the cavity determines the line width of the diode laser if the feedback is strong enough. Thus, line widths of a few $\mathrm{kHz}$ are feasible [2]. The cavity length can be controlled via PZT's to tune the diode laser over a modest range, typically a few $100 \mathrm{MHz}$. Thus, combination of the two concepts seems to be the consecutive step forward. In the approach by Patrick and Wieman [4] and later by Hayasaka [5] the 0th order output of a grating is coupled to an external cavity and from there fed back into the diode laser. The disadvantage of this system is, that the two feedback channels compete with each other. This results in a situation where stable operation is difficult to achieve.

\footnotetext{
${ }^{1}$ Electronic adress: Andreas.Wicht@uni-duesseldorf.de

${ }^{2}$ Electronic adress: rinkleff@ceres.amp.uni-hannover.de

In order to combine the advantages of the external cavity and the extended cavity diode laser setup we develop the grating enhanced external cavity diode laser setup (GEECDL), which was first described in a theoretical line width analysis by Belenov and co-workers [6]. This approach has also been investigated experimentally in [7] for two specific types of cavity geometries. In our work we systematically analyze various cavity geometries with respect to their performance as part of the new diode laser setup. We discuss the optimum cavity geometry and show results achieved with our experimental setup. We should mention that our theoretical investigation provides the means to select the proper cavity geometry and therewith complements the analysis by Belenov. As it turns out, a proper cavity design is essential for successful operation of a GEECDL.

\section{Basic idea and theory}

We start our description with the Littman-setup and replace the folded V-type cavity in fig. 1 by a retro-reflecting mirror. The grating disperses the incident ray into rays with different angles corresponding to different wave lengths. The ray of wave length $\lambda$ hits the hypothetical Littman-mirror under an angle $\alpha$ and is reflected under an angle $\beta=\alpha$. The reflected ray incident on the grating undergoes diffraction for a second time. The 1st order is then at an angle $\gamma$ with respect to the ray emitted by the laser diode. Only the rays at sufficiently small angles $\gamma$ are fed back and amplification at these wave lengths can take place.

We now replace the retro-reflecting mirror by the V-type cavity shown in fig. 1, so that becomes a function of the angle $\alpha, \beta=\beta(\alpha)$. The ray with wave length $\lambda$ is coupled into the cavity at the angle $\alpha$. After $n$ round trips it leaves the cavity under an angle $\beta_{n}$ and is fed back via 1 st order at an angle $\gamma_{n}$. As long as $\left|\gamma_{n}\right| \geq|\alpha|$ is fulfilled, the spectral selectivity of the setup is comparable to that of the corresponding Littrow-setup.

Applying the ABCD-formalism [8] we find that $\left|\gamma_{n}\right| \geq|\alpha|$ corresponds to the condition:

$\partial_{\alpha} \beta_{n}(\alpha) \notin[-2,0]$

where $n=0 \ldots N$ is giving the effective number of round trips in the cavity. Assuming that the finesse of our feedback cavity does not exceed $F \sim 150$ we limit our analysis to $N=25$ which is in good agreement with our experiments $(F \sim 50 \ldots 100)$. For the Littman-setup no round trips are performed, $n=0$, and $\alpha=\beta=\gamma / 2$ so that $\partial_{\alpha} \beta_{0}(\alpha)=1$.

If the condition (1) is not fulfilled the dispersion of the grating is partially canceled by the cavity possibly leading to unstable operation. Thus we have to to determine which cavity geometries provide stable operation. In our analysis symmetric and stable 


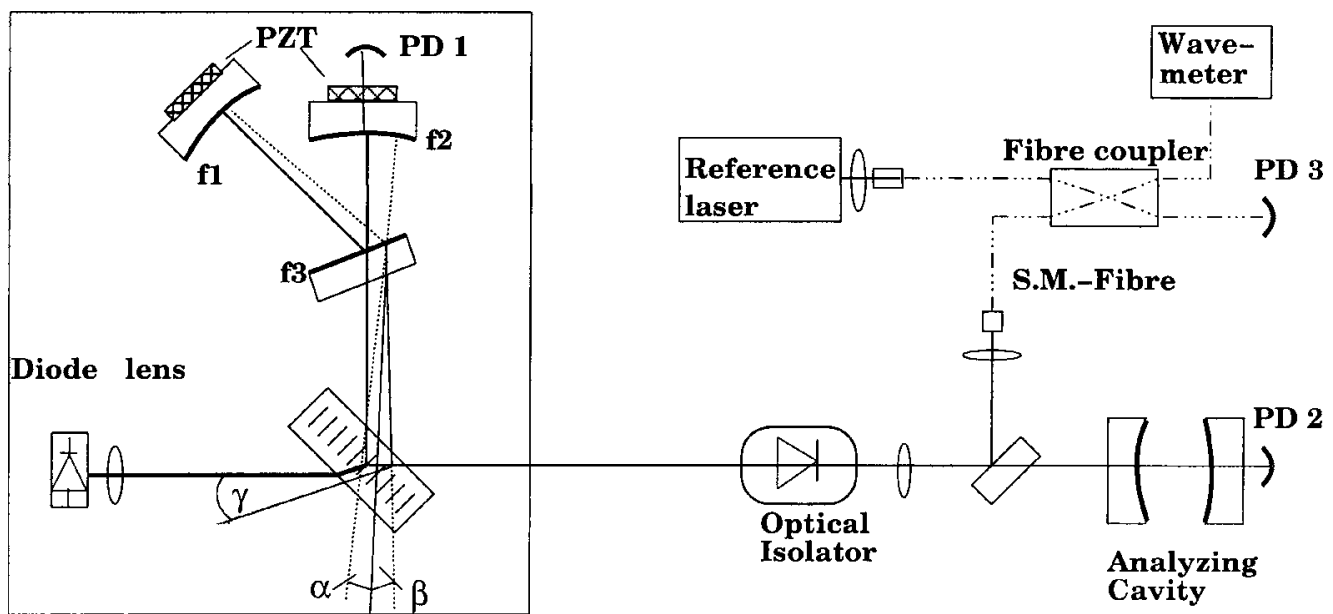

Fig. 1. Setup of the GEECDL: In the right part the analysis setup is shown. PD 1 (photo diode) is used for adjustments (laser threshold and alignment of the confocal cavity). PD 2 is used to check whether the GEECDL runs single mode. PD 3 detects the beat note of the GEECDL and a reference laser (diode laser with resonat optical feed back). With the wave meter and the signal from PD 2 the tuning ranges are investigated.

resonators are described by a single number, the geometry factor $\epsilon$.

$\epsilon=D /(4 f)$

where $D$ is given by the dimension of the cavity. $f$ is the focal length of the curved mirrors. In our analysis we always assume $f_{3}=\infty$, see fig. 1 . We consider the following classes: $(i) f_{1}=\infty$ and $f_{2}=f$, (ii) $f_{1}=f$ and $f_{2}=\infty$. For those $D$, equals twice the overall cavity length. The third case (iii) corresponds to $f_{1}=f_{2}=f$ and here $D$ equals the overall cavity length. In general, for the class of stable resonators $\epsilon$ ranges from $0 \ldots 1$ and $\epsilon=0, \epsilon=0.5$ and $\epsilon=1$ correspond to the planar, to the confocal and the semiconcentric geometry. For a specific geometry described by $\epsilon$ we calculate $\partial_{\alpha} \beta_{n}$ for $n=0 \ldots N$. All $\beta_{n}$ for $N=10$ are shown in fig. 2. We relax our condition (1) to account for the fact that it is impossible to exactly realize a specific geometry.

$\partial_{\alpha} \beta_{n}(\alpha) \notin[-1.9,-0.1]$.

This replacement provides the possibility to find potentially interesting geometry ranges $[\epsilon-\Delta \epsilon / 2, \epsilon+\Delta \epsilon / 2]$ rather then singular geometries. For all geometries within this range the related condition (3) is satisfied. These geometries therefore provide a spectral selectivity which is not significantly reduced with respect to the Littrow setup. Another important criterion is how well the mode of the beam emitted by the laser diode

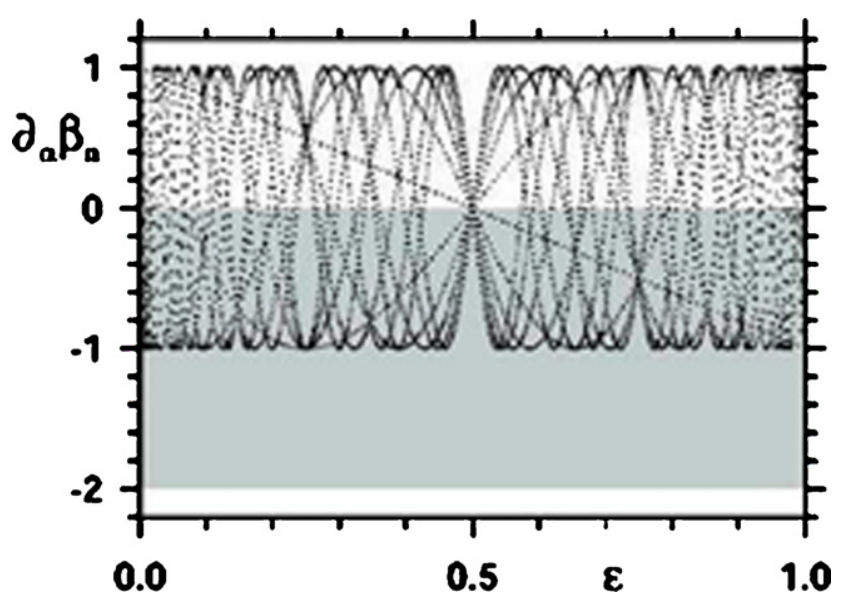

Fig. 2. The condition: $\partial_{\alpha} \beta_{n}(\alpha)$ vs. $\epsilon . n=0 \ldots 10$. The range of $\partial_{\alpha} \beta_{n}(\alpha)$ which has to be avoided is shown in gray. is matched to the cavity. If the beam of the laser diode is well collimated, it has a diameter of roughly $1 \mathrm{~mm}$, which is larger by a factor $\sim 10$ than the waist of the cavity fundamental mode. Hence we would have to use additional lenses to ensure efficient mode matching. We do not consider this as useful, since every surface will create additional feedback. Further, a complex mode matching lens system would require more space and therefore would reduce the mechanical stability of the setup.

\section{Setup and results}

In our experimental setup we investigated three different cavity geometries, all using planar input couplers, $f_{3}=\infty$ : the planar $\left(f_{1}=f_{2}=\infty, \epsilon=0\right)$, the semiconcentric $\left(f_{1}=\infty, f_{2}=f, \epsilon=\right.$ $0.5)$, and the confocal case $\left(f_{1}=f_{2}=f, \epsilon=1\right)$. The laser diode is an index guided single mode laser diode provided by Sacher Lasertechnik (SAL 850-50). The front facet of the laser diode is AR-coated showing a residual reflectivity of $R=3 \cdot 10^{-5}$ at the central wave length of $852 \mathrm{~nm}$. The uncoated laser diode is specified to emit $50 \mathrm{~mW}$ at $865 \mathrm{~nm}$. The beam is collimated by an AR-coated lens manufactured by Geltech (C390TM-B) with $N A=0.65$ and $f=2.75 \mathrm{~mm}$. The transmission grating used in our setup is a holographic grating manufactured by STEAG ETAOptik with a line density of $16001 / \mathrm{mm}$. The loss induced by the grating strongly depends on the polarization. For the polarization parallel to the plain of incidence a loss of $12 \%$ is measured whereas $30 \%$ is measured for the perpendicular orientation. This indicates that most of the loss is due to Fresnel reflection at the grating surfaces which are not AR-coated. $65 \%$ of the incident light is coupled into the 1 st order of diffraction. Typical power levels are $3.3 \mathrm{~mW}$ and $18.1 \mathrm{~mW}$ for 0 th and 1 st order output, respectively, corresponding to a diode current of $85 \mathrm{~mA}$. The input coupler, $f_{3}$, see fig. 1 , was specified to have $8 \%$ transmission under $45^{\circ}$ of incidence at $846 \mathrm{~nm}$. The end-mirrors, $f_{1}$ and $f_{2}$, of the cavity show a reflectivity $>99 \%$.

In absence of optical feedback the threshold current $\mathrm{I}_{t h}$ is determined to be $53.4 \mathrm{~mA}$. With feedback from the planar cavity this is reduced to $I_{t h}=30.0 \mathrm{~mA}$; the semiconcentric cavity shows $I_{t h}=30.3 \mathrm{~mA}$ whereas for the confocal geometry the threshold is as low as $24.1 \mathrm{~mA}$. This suggests that the most stable operation should be achievable with the confocal geometry. All threshold currents are measured at constant diode temperature $\left(13.3^{\circ} \mathrm{C}\right)$ and 


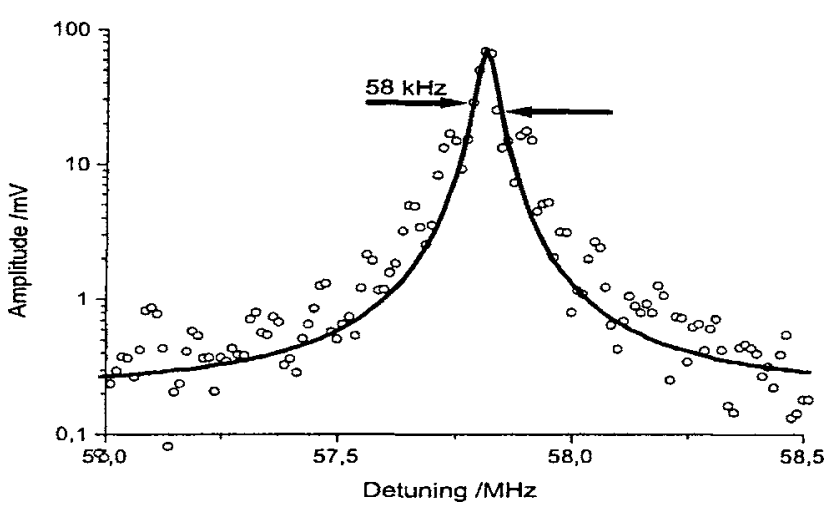

Fig. 3. Beat note signal between the GEECDL and the reference laser determines an upper limit for the short term line width of $58 \mathrm{kHz}$. Measured with an Agilent A4395 spectrum analyzer at a sweep time of $76.3 \mathrm{~ms}$ for 801 points in a $10 \mathrm{MHz}$ scan at a resolution bandwidth of $30 \mathrm{kHz}$.

constant wave length $(852 \mathrm{~nm})$. We then measure the continuous tuning range. In order to tune the wave length we control the length of the cavity with both PZTs shown in fig. 1 and simultaneously adjust the diode current. $4.4 \mathrm{GHz}, 19.6 \mathrm{GHz}$ and $45.1 \mathrm{GHz}$ continuous tuning range is achieved for the planar, semiconcentric, and confocal cavity geometry. The $45.1 \mathrm{GHz}$ and $19.6 \mathrm{GHz}$ are limited by the dynamic range of the PZTs. In an additional experiment we determine the overall tuning range for the confocal geometry to be $36.4 \mathrm{~nm}$ at constant diode temperature.

In a beat note experiment with a reference laser we estimate the upper line width of the GEECDL with the confocal cavity geometry, see fig. 3. The reference laser is a diode laser with optical feedback from an external cavity. The average short term line width of the beat note is $60 \mathrm{kHz}$ when the GEECDL is electronically frequency locked to the reference laser with a unity gain frequency of $\sim 400 \mathrm{~Hz}$, see fig. 4 . The residual frequency noise is dominated by low Fourier frequencies. Therefore, further improvement of the mechanical stability and the electronic feedback loop should reduce the noise significantly.

\section{Conclusion}

We presented theoretical and experimental investigations for diode lasers based on an advanced concept for optical feedback.

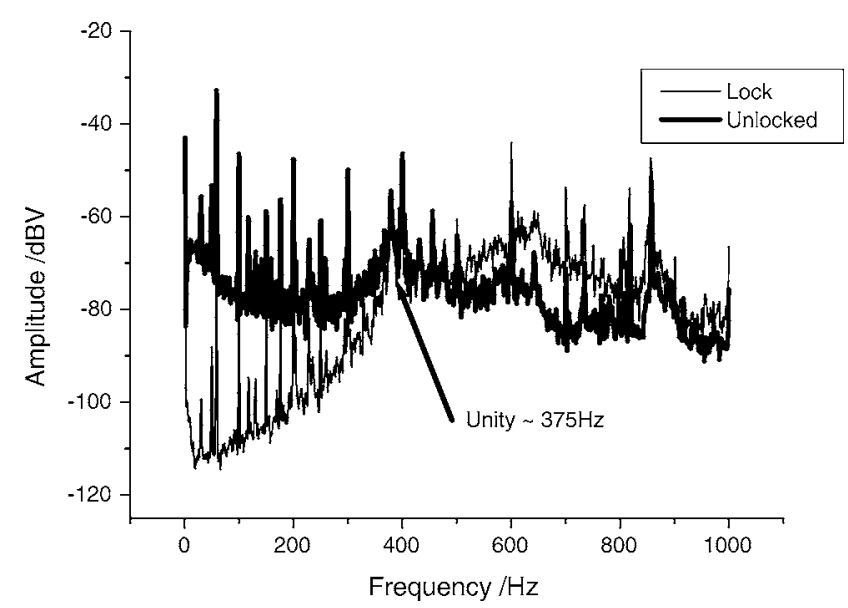

Fig. 4. Residual relative frequency noise between GEECDL and the reference laser in arbitrary units at low Fourier frequencies. The thinner line represents the GEECDL electronically locked to the reference laser while the thick line represents the open loop noise spectrum. Unity Gain is denoted.

The advantages of grating diode lasers and diode laser with resonant optical feed back from an external cavity were combined successfully. An over all tuning range of $36.4 \mathrm{~nm}$ and a continuous tuning range of $45.1 \mathrm{GHz}$ were demonstrated. We also successfully transfered the small line width known from diode lasers with resonant optical feedback to our setup, which showed a short term line width of at most $\sim 60 \mathrm{kHz}$.

\section{Acknowledgments}

This work was supported by the SFB407 of the Deutsche Forschungsgemeinschaft.

\section{References}

1. Fleming, M. W. and Mooradian, A., IEEE Quantum Electron. 17, 44 (1981).

2. Wieman, C. E. and Hollberg, L., Rev. Sci. Instrum. 62, 1 (1991).

3. Fox, R., Oates, C. and Hollberg, L., "Cavity enhanced Spectroscopy", (Edited by van Zee, R. D. and Looney, J. R.), (Academic Press, Amsterdam 2002) p. 1.

4. Patrick, H. and Wieman, C. E., Rev. Sci. Instrum. 62, 2593 (1991).

5. Hayasaka, K., Opt. Commun. 206, 401 (2002).

6. Belenov, É. M. et al., Sov. J. Quantum Electron. 13, 792 (1983).

7. Knaak, K.-M., Ph.D. Thesis, Universität Heidelberg (2000).

8. Siegmann, A. E., "Lasers", (University Science Books, Sauselito, CA, 1986). 